\title{
A case of reactivation of varicella-zoster virus after BNT162b2 vaccine second dose?
}

\author{
Luca Spiro Santovito ${ }^{1} \cdot$ Graziano Pinna ${ }^{1}$ (i)
}

Received: 12 July 2021 / Revised: 26 July 2021 / Accepted: 2 August 2021 / Published online: 14 August 2021

(c) The Author(s), under exclusive licence to Springer Nature Switzerland AG 2021

\begin{abstract}
We report a case of itchy papulovesicular rash consistent with varicella-zoster virus reactivation after Pfizer-BioNTech vaccine second dose administration. While there have been cases of varicella-zoster virus reactivation due to COVID-19 or COVID-19 vaccine inoculation in older individuals with pre-existing conditions, this case report describes the first case of varicella-zoster virus reactivation on a healthy, young male in the absence of pre-existing conditions. The mechanisms underlying varicella-zoster virus reactivation in patients with COVID-19 are unknown and should be further characterized.
\end{abstract}

Keywords Pfizer-BioNTech $\cdot$ COVID-19 vaccinations $\cdot$ Varicella-zoster virus $\cdot$ Shingles

\section{Introduction}

Pfizer-BioNTech vaccine is a nucleoside-modified RNA vaccine (BNT162b2), and it was the first vaccination approved by the US Food and Drug Administration (FDA), after an emergency use authorization, on December 11th, 2020 [1]. We know little about the long-term and rare adverse effects of COVID-19 vaccinations; thus, it is crucial to report side effects related to their administration to expand our knowledge on the safety profile of the vaccines and promote their use globally.

Here, we describe a case of itchy papulovesicular rash, which is consistent with shingles reactivation following Pfizer-BioNTech vaccine second dose. Cases of varicella-zoster virus reactivation due to COVID-19 and its vaccination have already been reported in older individuals with pre-existing conditions, including hypertension, cerebrovascular accident and bladder cancer, with hypertension and anti-neutrophilic cytoplasmic antibody-related glomerulonephritis, or with autoimmune inflammatory rheumatic disease [2-6]. This report is the first to describe a case

Responsible Editor: John Di Battista.

Graziano Pinna

gpinna@uic.edu; graziano_pinna@yahoo.com

1 Department of Psychiatry, The Psychiatric Institute, College of Medicine, University of Illinois Chicago (UIC), 1601 West Taylor Street, Chicago, IL 60612, USA of shingles reactivation on a healthy, young adult male in absence of pre-existing conditions.

The process that causes varicella-zoster reactivation is unknown, as well as the incidence of shingles after PfizerBioNTech vaccine inoculation. More studies are needed to better understand the association between the vaccine and the varicella-zoster virus reactivation.

\section{Case report}

The patient, a 27-year-old white male, received the first vaccination dose on March 16th, 2021 and the second dose on April 10th, 2021. Three days after, two small, itchy, pink papular rashes $(<2 \mathrm{~mm})$ started to develop on the volar aspect of his inner left forearm. Between April 13th and April 16th, the rash became itchier, and the lesions increased in size, reaching $15 \mathrm{~mm}$ in diameter, and in number, spreading to the entire left upper limb (Fig. 1A), and involving the left upper pectoral region, the left armpit, the left cubital hollow, the volar aspect of the left forearm, and the dorsal aspect of the second and third fingers.

Since the evolution of the symptoms and the related discomfort, the subject contacted his family doctor, who ruled out poison ivy, molluscum contagiosum, scabies, bug bites, and prescribed $40 \mathrm{mg}$ of prednisone, $25 \mathrm{mg}$ of hydroxyzine, and $2 \%$ mupirocin ointment. This treatment was started on the evening of April 16th, 2021. After 6 days of treatment, the subject experienced no apparent improvement in either 

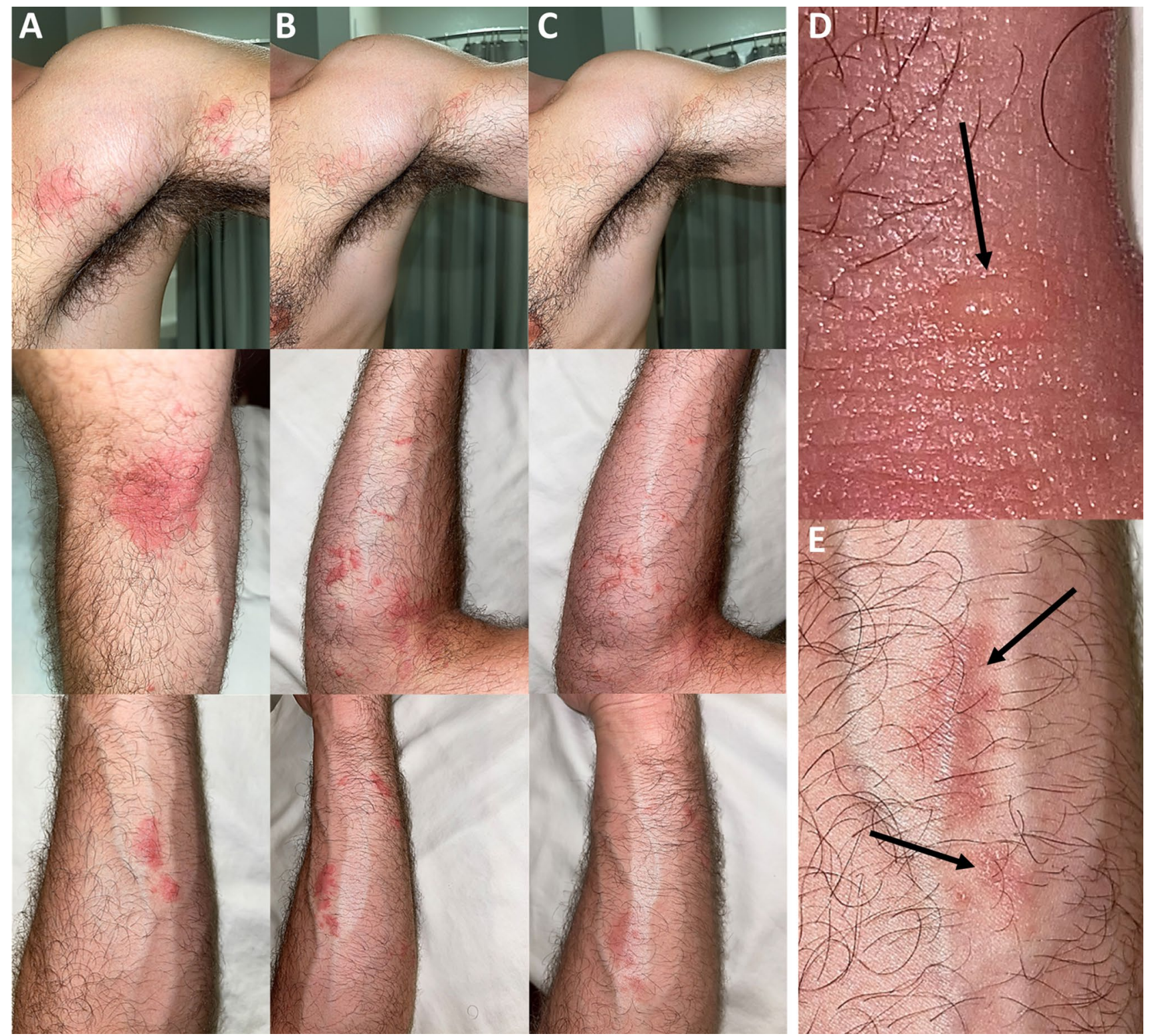

Fig. 1 Urticarial-like exanthema developed in papulo-vesicular before regressing with scar lesions. A. The rash started as urticariallike exanthema on April 16th. The left armpit and left upper pectoral region, the volar aspect of the left forearm and the left cubital hollow are shown in these pictures. B. On April 22nd, the rash started to spread, and the subject developed vesicular lesions, filled with serous exudates. C. Papularvesicular lesions started to regress and scar lesions developed from April 28th. D. Vesicular blisters (arrows) filled with serous exudates on the dorsal aspect of the left hand. E. Scar lesions, typical after shingles infection itchiness or skin rash morphology. Indeed, in the following days, the lesions worsened becoming itchier and vesicular, forming blisters filled with serous (Fig. 1B and D). The aspect of the rash recalled herpes zoster disease and, interestingly, the patient had chickenpox during his childhood when he was 6 years old. After 10 days of treatment without improvement and being concerned about the evolution of the rash, he contacted his doctor again, who prescribed an echocardiogram, which resulted normal. After 18 days (Fig. 1C), the papulovesicular rash started to regress, with scar lesions that replaced the vesicles and then, it completely disappeared after 30 days. Details of the lesions are shown in Fig. 1E.

\section{Varicella-zoster virus reactivation after BNT162b2 vaccine second dose}

COVID-19 has been associated with several skin manifestations, ranging from urticarial rash, morbilliform exanthema, papulovesicular exanthema to livedo reticularis and purpuric 
exanthema. Recently, Maia and colleagues [2] reported an increased number of varicella-zoster virus cases in Brazil and assumed an association with the COVID-19 pandemic.

Varicella-zoster virus belongs to the Herpesviridae family, and it is the causative agent of chickenpox in children and young adults, and shingles, mainly in adults. After the resolution of the primary infection, the virus remains latent in the ganglia of the dorsal roots of the cranial nerve. Stress or trauma, other than immunosuppression status, can reactivate the virus causing Herpes Zoster disease, also known as shingles.

The papulovesicular rash described in our report strongly recalls a varicella-zoster virus reactivation triggered by the second dose of the Pfizer-BioNTech vaccine. Consistent with the Varicella-zoster incubation period, which ranges from 1 to 5 days, the subject started to develop the rash 3 days after the vaccine injection. Also, the evolution of the rash is typical, starting with an urticarial-like papular rash, and becoming vesicular with blisters filled with serous exudates immediately after. Moreover, the lesions developed in the innervation territory of the roots of the left brachial plexus (from $\mathrm{C} 5$ to $\mathrm{T} 1$ dermatomes) that is in line with the typical shingles presentation, which follows a dermatomal distribution.

Although the absence of laboratory tests (varicella-zoster virus-specific IgM antibodies and/or PCR) and histological evaluation, which would confirm the diagnosis, the hypothesis of a varicella-zoster virus reactivation is conceivable. This is further supported by the varicella infection during the subject's childhood.

It is interesting to note that cases of varicella-zoster virus reactivation have already been described following vaccinations against Hepatitis A, influenza virus, rabies and Japanese encephalitis [3]. Recently, two cases of Herpes Zoster reactivation have been associated with COVID-19 vaccination; the first, a 78-year-old man with a history of hypertension, cerebrovascular accident and bladder cancer, after the inoculation of an inactivated COVID-19 vaccine [4]; the second, a 79-year-old man with a history of hypertension and anti-neutrophilic cytoplasmic antibody-related glomerulonephritis, after mRNA COVID-19 vaccination [5]. Moreover, Furer and colleagues recently described an interesting case series of varicella-zoster virus reactivation in 6 patients affected by autoimmune inflammatory rheumatic disease after Pfizer-BioNTech inoculation [6]. Thus, the present report constitutes the first case documented on a rare case of varicella-zoster virus reactivation on a healthy, young adult male without pre-existing conditions.

The Pfizer-BioNTech vaccine is the first approved vaccination by the FDA and administered in the USA and globally. The most common adverse effects are mild to moderate, and they can be local, involving the site of the injection (pain, swelling and redness) or systemic (chills, tiredness, and headache). In addition, diarrhea, nausea, vomiting, and deep tiredness have also been described.

We still know little about rare and long-term side effects related to COVID-19 vaccines and, even though the vaccination is crucial to stop spreading the virus, it is also important to monitor and report any possible adverse effect associated with the administration of the vaccines. Despite the impossibility of establishing the causal relation between our patient rash and the vaccine, it is reasonable to hypothesize that the vaccination-induced immune stress triggered the varicella-zoster virus reactivation, which is also consistent with the clusters of varicella-zoster virus reactivation already described in COVID-19 patients or in immune-compromised individuals.

The incidence of shingles after the COVID-19 vaccine inoculation is not known, more studies are needed to understand how the reactivation of varicella-zoster virus and the Pfizer-BioNTech vaccine are related.

Author contributions GP collected the data, LS analyzed the data, and LS and GP wrote the manuscript.

Funding The authors did not receive any funding for the present study.

\section{Declarations}

Conflict of interest On behalf of all authors, the corresponding author states that there is no conflict of interest or competing interests.

Informed consent The authors obtained the patient's written informed consent for the publication of the present case report.

\section{References}

1. https://www.fda.gov/emergency-preparedness-and-response/coron avirus-disease-2019-covid-19/pfizer-biontech-covid-19-vaccine

2. Maia MCF, Marques NP, de Lucena EHG, de Rezende LF, Martelli DRB, Marteli Junior H. Increased number of Herpes Zoster cases in Brazil related to the COVID-19 pandemic. Int J Infect Dis. 2021;104:732-3.

3. Walter R, Hartmann K, Fleisch F, Reinhart WH, Kuhn M. Reactivation of herpesvirus infections after vaccinations? The Lancet. 1999;353:810. https://doi.org/10.1016/S0140-6736(99)00623-6

4. Bostan E, Yalici-Armagan B. Herpes zoster following inactivated COVID-19 vaccine: a coexistence or coincidence? J Cosmet Dermatol. 2021;00:1-2. https://doi.org/10.1111/jocd.14035.

5. Eid E, Abdullah L, Kurban M, Abbas O. Herpes Zoster emergence following mRNA COVID-19 vaccine. J Med Virol. 2021. https:// doi.org/10.1002/jmv.27036.

6. Furer V, Zisman D, Kibari A, Rimar D, Paran Y, Elkayam O. Herpes zoster following BNT162b2 mRNA COVID-19 vaccination in patients with autoimmune inflammatory rheumatic diseases: a case series. Rheumatology. 2021. https://doi.org/10.1093/rheum atology/keab345.

Publisher's Note Springer Nature remains neutral with regard to jurisdictional claims in published maps and institutional affiliations. 